\title{
Interventions to Safeguard System Effectiveness During Periods of Emergency Department Crowding
}

Keith E. Kocher, MD, MPH, Steven A. Shane, MD, MS, Arjun K. Venkatesh, MD, MBA, Dominik Aronsky, MD, PhD, Brent R. Asplin, MD, MPH, and Niels K. Rathlev, MD

\begin{abstract}
This article summarizes the proceedings of a breakout session, "Interventions to Safeguard System Effectiveness," at the 2011 Academic Emergency Medicine consensus conference, "Interventions to Assure Quality in the Crowded Emergency Department." Key definitions fundamental to understanding the effectiveness of emergency care during periods of emergency department (ED) crowding are outlined. Next, a proposed research agenda to evaluate interventions directed at improving emergency care effectiveness is outlined, and the paper concludes with a prioritization of those interventions based on breakout session participant discussion and evaluation.
\end{abstract}

ACADEMIC EMERGENCY MEDICINE 2011; 18:1313-1317 (c) 2011 by the Society for Academic Emergency Medicine

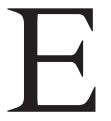
ffectiveness of care is a component of what the Institute of Medicine defines as one of the six core values for all health care services and is critical to maintaining health care quality. ${ }^{1}$ A working definition of effectiveness is essential to a focused emergency care research agenda. For the purposes of this consensus conference, we defined effectiveness as the delivery of evidence-based, guideline-concordant care. To date there has been limited study of the effect of emergency department (ED) crowding on effectiveness of care, despite the importance of this domain to quality care. ${ }^{2}$ A recent study demonstrated a relationship between escalating ED crowding and decreasing likelihood of adherence to clinical guidelines with delivery of corticosteroid treatment and documentation of severity scores in pediatric patients presenting with asthma. ${ }^{3}$ Unfortunately, this analysis is only one of a few that have shown a direct relationship between ED crowding and delivery of guideline-concordant care. Other studies, for example, have evaluated the relationship between crowding and timing of antibiotics for pneumonia ${ }^{4,5}$ and between crowding and thrombolysis ${ }^{6}$

From the Department of Emergency Medicine, University of Michigan (KEK), Ann Arbor, MI; St. Rose Dominican Hospitals and Children's Hospital of Nevada (SAS), Henderson and Las Vegas, NV; Brigham and Women's Hospital-Massachusetts General Hospital-Harvard Affiliated Emergency Medicine Residency (AKV), Boston, MA; the Departments of Biomedical Informatics \& Emergency Care, School of Medicine, Vanderbilt University (DA), Nashville, TN; Fairview Medical Group (BRA), St. Paul, MN; and the Department of Emergency Medicine, Baystate Medical Center and Tufts University School of Medicine (NKR), Springfield, MA.

Received July 15, 2011; revision received August 12, 2011; accepted August 12, 2011

Participants: Dominik Aronsky, Brent Asplin, Kevin Baumlin, Christopher Beach, Steven Bernstein, Michelle Blanda, Joseph Bushra, Linda Davis-Moon, Sarang Deo, Susan Duffy, Eric Goldlust, Matthew Gratton, David Griffen, Leon Haley, Jr., Kendall Hall, Thomas Riisgaard Hansen, Keith Kocher, Mark McClelland, Brad Morrison, Elaine Rabin, Niels Rathlev, John Rogers, Richard Ruddy, Steven Shane, Robert Sherwin, Inger Sondergaard, Olan Soremekun, Susan Stern, Seth Trueger, Arjun Venkatesh, and Andrew Zinkel.

Funding for this conference was made possible (in part) by 1R13HS020139-01 from the Agency for Healthcare Research and Quality (AHRQ). The views expressed in written conference materials or publications and by speakers and moderators do not necessarily reflect the official policies of the Department of Health and Human Services, nor does mention of trade names, commercial practices, or organizations imply endorsement by the U.S. Government. This issue of Academic Emergency Medicine is funded by the Robert Wood Johnson Foundation.

The authors have no potential conflicts of interest to disclose.

Supervising Editor: James Miner, MD.

Address for correspondence and reprints: Keith E. Kocher, MD, MPH; e-mail: kkocher@umich.edu. 
or percutaneous intervention ${ }^{7,8}$ for acute myocardial infarction.

Emergency department crowding has been linked to patient morbidity and mortality across a wide spectrum of clinical conditions over the past decade, including cardiac and critical care populations..$^{8-12}$ One potential explanation for poorer outcomes is a decrease in clinical effectiveness during periods of crowding. ${ }^{2}$ If we are to mitigate the effects of crowding, we will need to identify those interventions that will help us make the right decisions when the ED and providers are overloaded. Our hypothesis was that ED crowding exposes specific vulnerabilities that impair the ability to maintain emergency care effectiveness. We describe a research agenda designed to evaluate proposed interventions to maintain effectiveness of emergency care during these vulnerable periods. We frame these interventions in the traditional input-throughput-output model of ED crowding. ${ }^{13}$

\section{INPUT: PROLONGED WAIT TIMES}

As EDs become more crowded, overall length of stay grows, waiting rooms swell, and patients spend more time waiting to be seen by a provider. This waiting period creates a unique obstacle to prompt clinical evaluation and appropriate care delivery. The primary challenge in capacity-constrained, crowded EDs is to maintain effective emergency care that identifies patients requiring time-sensitive, guideline-based care. Potential interventions to address this challenge involve strategies to facilitate the early initiation of diagnosis and treatment.

The consensus conference breakout group identified interventions directed at initiating diagnostic and treatment protocols in the waiting room as an opportunity to improve effectiveness. For example, nurses or physician extenders could begin evaluation and treatment so that appropriate care has been initiated before evaluation by the senior provider. Furthermore, creating interventions for waiting room assessment can facilitate earlier administration of analgesics or other medications for which timely administration is considered an important element of effective care. Another proposed group of interventions identified were the potential to have waiting patients complete questionnaires (medications, allergies, medical history, etc.) to facilitate the likelihood of gathering the accurate and comprehensive information needed to deliver effective emergency care. The breakout group was careful to note that studies intended to measure improvements in effectiveness from these types of interventions would need to use clinically meaningful outcomes linked to guideline adherence, such as administration of aspirin in acute myocardial infarction or timely thrombolysis for acute ischemic stroke.

\section{THROUGHPUT: COGNITIVE OVERLOAD}

As EDs become more crowded, individual providers face an exponential increase in the number of decisions that need to be made, decreasing the time available to make each decision. ED crowding therefore increases "decision density"14 at the same time that providers are more likely to be interrupted. These phenomena create conditions of cognitive overload, a term used to describe the background information and bits of memory a provider must increasingly bring to bear on critical decisions. Cognitive overload impairs provider performance and can result in poorer decision-making, errors, and adverse events. ${ }^{2,14,15}$ The breakout group identified potential interventions that may combat the challenges presented by cognitive overload, including: 1) team training, 2) electronic alerts, 3) care plans, 4) protocols for specific diseases, and 5) information technology (IT) systems.

Medical team training is focused on teaching the coordination of tasks and communication with all providers (e.g., providers, nurses, and technicians) in a particular setting or department, with the shared goal of improving group performance and reducing errors during care delivery. ${ }^{16}$ This approach stems from the military and aviation industries. A recent review of medical team training describes research demonstrating an association between teamwork and reduced medical errors. ${ }^{17}$ Perceptions of staff attitude toward teamwork are associated with improved quality and improved staff well-being, and additionally, good team behavior is related to improved clinical performance. ${ }^{17}$ Despite some evidence supporting team training, ${ }^{18}$ actually demonstrating that the process of team building as an intervention can result in improved outcome has not been done in the medical clinical arena, let alone provide improved effectiveness in the crowded ED. However, team training may have hope of improving adherence to clinical guidelines, care plans, and algorithms with potential improvements in medical error rates and patient morbidity and mortality during periods of ED crowding.

Electronic alerts may provide another opportunity to ensure effective care during crowded periods by providing real-time decision support. Many health IT systems already have the capacity to provide this, but there is a paucity of literature supporting the use of alerts in any clinical setting, particularly the ED. Most of the existing literature has focused on alerts built into prescribing software in the ambulatory clinic setting to warn providers of adverse effects or remind them to order baseline labs for specific medications. ${ }^{19,20}$ Using newer-generation alert platforms that may include task prioritization levels could facilitate a variety of support models, such as the delivery of standard medications (e.g., corticosteroids to an asthma patients), the ordering of guideline-recommended tests (e.g., chest computed tomography scan based on the Wells criteria), and compliance with publicly reported performance measures. $^{21}$ The breakout group identified one concern that relates to the potential for "alert fatigue" when alerts are overused or cannot be prioritized. This situation could lead providers to ignoring or overriding them, defeating the purpose of the alert.

Care plans targeted to specific, complex patient situations may also represent a set of interventions with the potential to improve effectiveness of care delivery during periods of crowding. These care plans might allow providers to focus on the provision of the essentials of necessary and guideline-concordant care during 
periods of crowding. The more closely these care plans are tied to the unique needs of individual patients, the more useful they are likely to be during periods of crowding, with the caveat that they also create the potential for anchoring bias and could potentially decrease the likelihood that providers will detect important variations from prior presentations.

Protocols for specific diseases have been shown to improve adherence to recommended care, decrease variability, and improve outcomes for high-risk, timesensitive conditions such as stroke, acute myocardial infarction, trauma, and sepsis. ${ }^{22}$ Perhaps the use of protocols could be expanded for application in highervolume, lower-acuity illnesses (e.g., asthma, psychiatric presentations, venous thromboembolic disease), which may have more effect during periods of crowding. The breakout group identified a spectrum of research hypotheses with respect to condition-specific protocols, including whether such protocols should be studied for general utilization as a means to improve effectiveness or whether they should only be used at times of ED crowding.

While sometimes considered a panacea by early adopters, health IT systems need new designs and process engineering. A review of health IT system studies, particularly those with clinical decision support and computerized provider order entry, have been demonstrated at certain institutions to positively affect physician behavior, resulting in reduced medication errors and adverse drug effects, improved adherence to guidelines, and reduced costs. ${ }^{23,24}$ These studies have been conducted predominantly in ambulatory care and inpatient settings and have not been applied to measuring effectiveness in the ED. There are concerns regarding the cumbersome task of data entry, which can involve keyboarding or dictating. Different data entry methods should be compared in regard to provider productivity and patient flow in the ED, particularly during periods of high volume. Furthermore, unintended consequences of implementing health IT systems include taking providers away from the bedside and decreasing direct verbal communication between members of the ED team. The impact these factors have on effectiveness during crowding in the ED is unknown and deserves investigation.

\section{OUTPUT: PERIODS OF BOARDING}

Periods of high occupancy often result in the practice of boarding, where admitted patients remain in the ED awaiting placement in inpatient hospital beds, making emergency care effectiveness vulnerable to breakdowns due to increased handoffs and delays in inpatient diagnostic or treatment plans. The challenge to maintaining effectiveness is to continue the delivery of guidelineconcordant care during periods of prolonged patient boarding time and frequent provider hand-offs. Potential interventions include checklists and improvements in care coordination within patient units, consultants, and admitting providers.

Checklists have been employed in the aviation industry ${ }^{25}$ to decrease errors of omission and increase reliability and have also received recent popular attention regarding their application to the medical care setting. ${ }^{26}$ Checklists have been shown to be effective in certain medical applications such as surgical site infection reduction and reduced central venous catheter complications. ${ }^{27}$ During crowded periods, patients are subject to more hand-offs, and each provider sign-out represents an opportunity for the transfer of critical information to be inadvertently omitted between physicians, nurses, and other providers. Checklists such as the SBAR (Situation-Background-Assessment-Recommendation) technique ${ }^{28}$ might allow for a focused means of communicating this information to ensure continued effectiveness of care under stressed ED conditions. Checklists could also be employed before and during completion of technical tasks to maintain effectiveness of care during ED crowding.

Another potential avenue of exploration is to improve care coordination with inpatient units, consultants, and admitting providers during periods of ED crowding. Perhaps during these critical periods, inpatient physicians would agree to expedite the admission or consultative process to maintain a seamless transition of care from the ED to the inpatient setting. There is some patient safety evidence supporting the strategy of immediate inpatient bedding under conditions of high ED occupancy, ${ }^{29}$ but this has not been specifically studied for maintaining effectiveness of care delivery. The challenge may be that these interventions could create crowded conditions in other areas of the hospital, which does not ultimately improve overall system effectiveness.

\section{OUTPUT: DISCHARGE PROCESS}

More than $80 \%$ of patients evaluated in the ED are discharged home. ${ }^{30}$ During periods of crowding, ensuring an effective outpatient care management plan may become more difficult, as providers potentially rush to disposition patients. Follow-up care and discharge instructions, a challenge even during noncrowded periods, ${ }^{31}$ may therefore deteriorate accordingly. Unlike admitted patients, discharged patients have no provider continuously overseeing their care. As a result, unforeseen outcomes in this group, coupled with an inability to access the timely outpatient or referral care, could result in negative patient outcomes. Potential interventions identified by the breakout group to improve discharges include standardization in the discharge process and telephone follow-up.

The discharge process is a critical transition in the course of the ED visit where important information and instructions are conveyed. During periods of crowding, this process may become disrupted or disjointed. Reducing the variability in discharge practices may improve the effectiveness of care during ED crowding. The use of standardized discharge instructions, prepared discharge instructions in multiple languages, structured patient comprehension assessment tools, and the use of "discharge teams" of nurses and other support staff were identified as methods that could enhance discharge practices. Such changes may improve the effectiveness of care due to better patient adherence to recommended aftercare instructions, such 
as prescription medication use, follow up visits, and reasons for return to the ED.

Another intervention to consider that might improve effectiveness is telephone follow-ups. Telephone care management has been shown to be cost-effective in managing long-term health care in the outpatient setting by reducing medical expenses and health care utilization, ${ }^{32}$ but has not been rigorously evaluated in the ED setting to address effectiveness of care. Telephone follow-up has been shown to be a viable means of communication for contacting discharged ED patients, however. ${ }^{33}$ After especially crowded ED shifts, contacting discharged patients to reinforce instructions and follow-up recommendations could increase the quality and effectiveness of care. Such an intervention could improve the transition of care to the patient's primary care physician or potentially lead to reduced ED revisits and improved medication adherence.

\section{PRIORITIZED RESEARCH AGENDA}

A variation on the nominal group technique developed by Delbecq and Van de Ven ${ }^{34}$ was used to prioritize the research interventions proposed for safeguarding system effectiveness during ED crowding. The group planning committee generated the research questions and potential interventions before the consensus conference. These were published in the meeting guide that was distributed to participants on the day of the meeting. During the breakout session, the facilitator presented sequentially each of the proposed interventions, and open discussion was solicited. A list of potential interventions was generated and organized based on modifications to the originally proposed categories for discussion. Each breakout group participant was given three votes to assess the relative importance of the various interventions proposed for study. There was no specification limiting participants to a single vote per intervention. The total number of votes for each intervention was tallied to determine the final ranking of research priorities.

The following interventions were thought to hold the most promise for study in maintaining effectiveness of emergency care during periods of ED crowding:

1. Electronic alerts (14 votes).

2. IT systems (12 votes).

3. Checklists (11 votes).

4. Protocols for specific diseases (11 votes).

5. Standardization in the discharge process $(10$ votes).

6. Team training (9 votes).

7. Early initiation of treatment (8 votes).

8. Telephone follow-up (7 votes).

9. Care plans (2 votes).

10. Improvements in care coordination with inpatient units, consultants, and admitting providers $(0$ votes).

\section{LIMITATIONS}

The prioritized research agenda represents the opinions of a small group of interested participants based on a breakout session discussion. The interventions considered were taken from a list generated by a series of meetings and discussions held among the breakout session organizers and published in the consensus conference's guide. Therefore, these proposed interventions may not be fully representative of all interventions to consider in maintaining effectiveness of emergency care during periods of crowding. There may be additional interventions that hold promise in mitigating the effects of ED crowding on effective care. In addition, the relative merits of the research priorities have not been evaluated scientifically, and it is possible that lower-ranked research priorities may ultimately be more useful for improving the effectiveness of emergency care during periods of crowding.

\section{CONCLUSIONS}

Emergency department crowding is an inevitable aspect of emergency care delivery. Maintaining effectiveness of care during periods of ED crowding is critical to ensuring quality emergency care. There are specific points of vulnerability that can occur throughout the time course of care during a patient's progression through the ED, including prolonged wait times, cognitive overload, boarding, and the discharge process. We have presented a prioritized research agenda aimed at studying interventions that hold promise at mitigating the effects of crowding on our ability to deliver evidence-based and guideline-concordant care to our patients.

The authors acknowledge the "Interventions to Safeguard System Effectiveness" breakout session participants from the 2011 Academic Emergency Medicine Consensus Conference, "Interventions to Assure Quality in the Crowded Emergency Department."

\section{References}

1. Institute of Medicine. Crossing the Quality Chasm: A New Health System for the 21st Century. Washington, DC: National Academies Press, 2001.

2. Pines JM, McCarthy ML. The crowding-effectiveness link: it doesn't matter how fast we deliver care if we don't deliver it right. Ann Emerg Med. 2011; $57: 201-2$

3. Sills MR, Fairclough D, Ranade D, Kahn MG. Emergency department crowding is associated with decreased quality of care for children with acute asthma. Ann Emerg Med. 2011; 57:191-200.

4. Fee C, Weber EJ, Maak CA, Bacchetti P. Effect of emergency department crowding on time to antibiotics in patients admitted with community-acquired pneumonia. Ann Emerg Med. 2007; 50:501-9.

5. Pines JM, Localio AR, Hollander JE, et al. The impact of emergency department crowding measures on time to antibiotics for patients with community-acquired pneumonia. Ann Emerg Med. 2007; 50:510-6.

6. Schull MJ, Vermeulen M, Slaughter G, Morrison L, Daly P. Emergency department crowding and thrombolysis delays in acute myocardial infarction. Ann Emerg Med. 2004; 44:577-85. 
7. Pines JM, Hollander JE, Localio AR, Metlay JP. The association between emergency department crowding and hospital performance on antibiotic timing for pneumonia and percutaneous intervention for myocardial infarction. Acad Emerg Med. 2006; 13:873-8.

8. Shen YC, Hsia RY. Association between ambulance diversion and survival among patients with acute myocardial infarction. JAMA. 2011; 305:2440-7.

9. Pines JM, Pollack CV, Diercks DB, Chang AM, Shofer FS, Hollander JE. The association between emergency department crowding and adverse cardiovascular outcomes in patients with chest pain. Acad Emerg Med. 2009; 16:617-25.

10. Richardson DB. Increase in patient mortality at 10 days associated with emergency department overcrowding. Med J Austral. 2006; 184:213-6.

11. Sprivulis PC, Da Silva JA, Jacobs IG, Frazer ARL, Jelinek GA. The association between hospital overcrowding and mortality among patients admitted via Western Australian emergency departments. Med J Austral. 2006; 184:208-12.

12. Guttmann A, Schull MJ, Vermeulen MJ, Stukel TA. Association between waiting times and short term mortality and hospital admission after departure from emergency department: population based cohort study from Ontario, Canada. BMJ. 2011; 342:d2983.

13. Asplin BR, Magid DJ, Rhodes KV, Solberg LI, Lurie $\mathrm{N}$, Camargo CA Jr. A conceptual model of emergency department crowding. Ann Emerg Med. 2003; 42:173-80.

14. Croskerry P, Sinclair D. Emergency medicine: a practice prone to error? Can J Emerg Med. 2001; $3: 271-6$.

15. Wears RL, Perry SJ. Human factors and ergonomics in the emergency department. Ann Emerg Med. 2002; 40:206-12.

16. Baker DP, Gustafson S, Beaubien JM, Salas E, Barach P. Medical team training programs in health care. In: Advances in Patient Safety: From Research to Implementation. Rockville, MD: Agency for Healthcare Research and Quality, 2005. Available at: http://aptima.com/publications/2005_Baker_Gustafson_ Beaubien_Salas_Barach.pdf. Accessed Sep 12, 2011.

17. Manser T. Teamwork and patient safety in dynamic domains of healthcare: a review of the literature. Acta Anaesthesiologica Scand. 2009; 53:143-51.

18. Morey JC, Simon R, Jay GD, et al. Error reduction and performance improvement in the emergency department through formal teamwork training: evaluation results of the MedTeams project. Health Serv Res. 2002; 37:1553-81.

19. Lo HG, Matheny ME, Seger DL, Bates DW, Gandhi TK. Impact of non-interruptive medication laboratory monitoring alerts in ambulatory care. J Am Med Informat Assoc. 2009; 16:66-71.

20. Weingart SN, Simchowitz B, Padolsky H, et al. An empirical model to estimate the potential impact of medication safety alerts on patient safety, health care utilization, and cost in ambulatory care. Arch Intern Med. 2009; 169:1465-73.

21. Niemi K, Geary S, Quinn B, Larrabee M, Brown K. Implementation and evaluation of electronic clinical decision support for compliance with pneumonia and heart failure quality indicators. Am J HealthSyst Pharm. 2009; 66:389-97.

22. Larsen GY, Mecham N, Greenberg R. An emergency department septic shock protocol and care guideline for children initiated at triage. Pediatrics. 2011; 127:e1585-92.

23. Shekelle PG, Morton SC, Keeler EB. Costs and benefits of health information technology. In: Evidence Report/Technology Assessment No. 132, Rockville, MD: Agency for Healthcare Research and Quality, 2006. Available at: http://www.ncbi.nlm.nih.gov/ books/NBK37988/. Accessed Sep 12, 2011.

24. Jamal A, McKenzie K, Clark M. The impact of health information technology on the quality of medical and health care: a systematic review. HIM J. 2009; 38:26-37.

25. Helmreich RL. On error management: lessons from aviation. BMJ. 2000; 320:781-5.

26. Gawande A. The Checklist Manifesto: How to Get Things Right. New York, NY: Metropolitan Books, 2009.

27. Pronovost P, Needham D, Berenholtz S, et al. An intervention to decrease catheter-related bloodstream infections in the ICU. NEJM. 2006; 355:272532.

28. Beckett CD, Kipnis G. Collaborative communication: integrating SBAR to improve quality/patient safety outcomes. J Healthc Qual. 2009; 31:19-28.

29. Viccellio A, Santora C, Singer AJ, Thode HC Jr, Henry MC. The association between transfer of emergency department boarders to inpatient hallways and mortality: a 4-year experience. Ann Emerg Med. 2009; 54:487-91.

30. Niska R, Bhuiya F, Xu J. National Hospital Ambulatory Medical Care Survey: 2007 Emergency Department Summary. Hyattsville, MD: National Center for Health Statistics, 2010.

31. Engel KG, Heisler M, Smith DM, Robinson $\mathrm{CH}$, Forman JH, Ubel PA. Patient comprehension of emergency department care and instructions: are patients aware of when they do not understand? Ann Emerg Med. 2009; 53:454-61.

32. Wennberg DE, Marr A, Lang L, O'Malley S, Bennett G. A randomized trial of a telephone caremanagement strategy. NEJM. 2010; 363:1245-55.

33. Goldman RD, Mehrotra S, Pinto TR, Mounstephen W. Follow-up after a pediatric emergency department visit: telephone versus e-mail? Pediatrics. 2004; 114:988-91.

34. Delbecq AL, Van de Ven AH. A group process model for problem identification and program planning. J Appl Behav Sci. 1971; 7:466-92. 